\title{
La fiction à l'épreuve du grotesque dans La Paranoïa de Rafael Spregelburd
}

La ficción como marco para evaluar lo grotesco en La Paranoia de Rafael Spregelburd The fiction to the test of grotesque in La Paranö̈a of Rafael Spregelburd

Nina Jambrina*

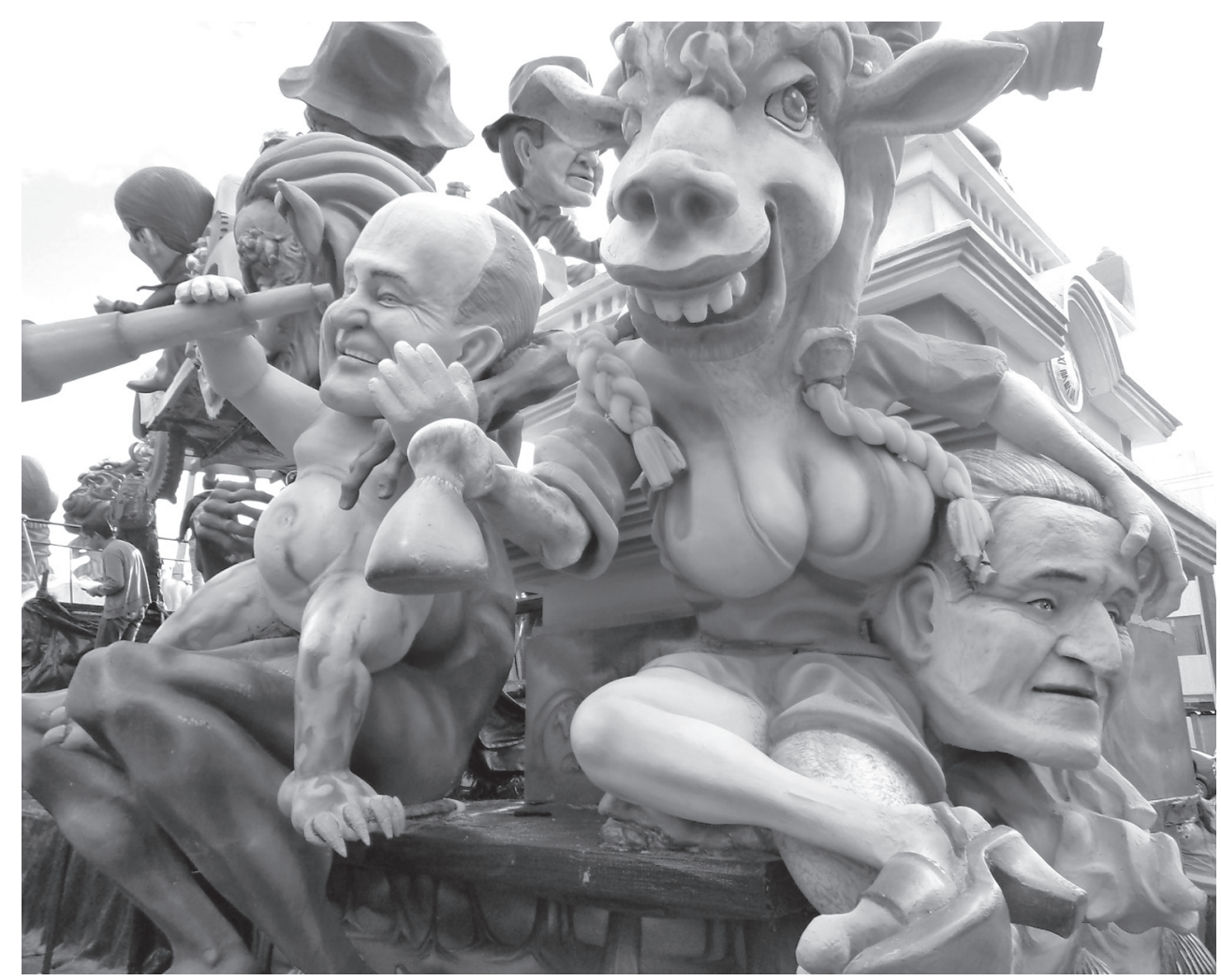

Citar este artículo como: Jambrina, N. (2017). La fiction à l'épreuve du grotesque dans La Paranö̈a de Rafael Spregelburd. Revista Papeles, 9(18), 34-44.

Fecha de recibido: octubre 5 de 2017.

Fecha de aceptación: noviembre 10 de 2017.

* Doctora en Estudios Teatrales, Nina Jambrina sustentó en 2017 una tesis titulada Política del Juego. Los dispositivos lúdicos en las dramaturgias latinoamericanas contemporáneas (Fabio Rubiano, Rafael Spregelburd y Gabriel Calderón). Hace parte del laboratorio LLA-CREATIS y es monitora en los departamentos de Artes vivas y Estudios Hispánicos e Hispanoamericanos de la Universidad Jean Jaurès - Toulouse II. Es miembro del comité lingüístico español de la Maison Antoine Vitez (Centro Internacional de Traducción Teatral). Correo electrónico: ninajambrina@hotmail.fr 


\title{
Résumé
}

Parmi les différents comiques que l'on connait, le grotesque tient une place à part pour relever à la fois du rire et de l'angoisse, du plaisir et du vertige. Il est le régime de l'invraisemblance, du contradictoire, de l'étrangeté mettant à l'épreuve ce qui nous est familier. Dans ce sens, la perspective grotesque semble particulièrement adaptée pour aborder les défis d'interprétation posés par de nombreuses œuvres contemporaines. La Paranoïa, écrite en 2009 par le dramaturge argentin Rafael Spregelburd, en est un exemple privilégié. La pièce met en œuvre un univers grotesque pour questionner la fabrique de la fiction elle-même et les habitudes de réception que nous en avons.

Mots clés: paranoïa, théâtre, grotesque.

\section{Resumen}

Entre los distintos cómicos que conocemos, el grotesco tiene un lugar aparte, en la medida en que tiene que ver a la vez con la risa y la angustia, el placer y el vértigo. Trabaja a la disposición de la inverosimilitud, la contracción, la extrañez, poniendo a prueba todo lo que nos es familiar. En este sentido, la perspectiva grotesca parece particularmente adecuada para estudiar los desafíos interpretativos que nos plantean muchas de las obras contemporáneas. La Paranoia, escrita en el 2009 por el dramaturgo argentino Rafael Spregelburd, es un ejemplo privilegiado. La obra construye un universo grotesco que interroga la fábrica de la ficción misma y las costumbres de recepción que tenemos de ella.

Palabras clave: paranoia, grotesco, teatro.

\begin{abstract}
Among the various comics we know, the grotesque holds a place apart for both laughter and anxiety, pleasure and vertigo. It is the regime of improbability, contradictory, strangeness putting to the test what is familiar to us. In this sense, the grotesque perspetive seems particularly suited to address the interpretive challenges posed by many contemporary works. The Paranoia, written in 2009 by the argentine playwright Rafael Spregelburd, is a prime example. The play implements a grotesque universe to question the fabric of fiction itself and the reception habits that we have of it.
\end{abstract}

Key words: paranoia, theater, grotesque.

\section{Introduction}

Qu'est que peut nous apporter aujourd'hui le grotesque? En quoi participe-t-il d'une meilleure compréhension des écritures contemporaines? Tour à tour synonyme de monstrueux, bizarre, caricatural, burlesque ou encore ridicule, la notion de grotesque aussi datée que résistante à toute définition pérenne semble correspondre à des esthétiques du mouvement et de la multiplicité si chères à la création au XXI siècle.

Dans ce sens, Rafael Spregelburd ${ }^{1}$ auteur, metteur en scène et acteur argentin déploie

1 Rafael Spregelburd est né à Buenos Aires en 1970 et y réside toujours aujourd'hui. Metteur en scène argentin largement reconnu dans son pays et un des plus lus de sa génération au niveau international. 
ses œuvres dans une perspective que l'on pourrait facilement qualifiée de grotesque. Actif depuis le début des années quatre-vingtdix, le théâtre indépendant argentin vivant alors une période de grande effervescence et de rupture postdictature, son théâtre atypique reste jusqu'à aujourd'hui un des plus suivis par le public et la critique dans le pays. Il compte plus d'une trentaine d'œuvres traduites dans plusieurs langues et a reçu un nombre conséquent de prix. La forme souvent démesurée et excessive de ces textes tend à se confondre avec un corps dont les intrigues démultipliables et échangeables à merci se répondent en échos tel un organisme vivant. La matière fictionnelle convoquée travaille, elle, à un questionnement permanant de l'identité de ses personnages et du genre artistique de ses situations. Son œuvre, L'Héptalogie de Hieronymus Bosch écrite entre 1996 et 2009 en est un exemple frappant.

Cette Héptalogie nait de la rencontre de Rafael Spregelburd avec la table-tableau de Jérôme Bosch exposé au Prado Les Sept Péchés capitaux et les Quatre Dernières Étapes humaines (1485). Le dramaturge se passionne alors pour l'univers visuel et symbolique du peintre Bosch à la fois riche et inquiétant, qui semble signer la fin d'une époque, le Moyen-âge, et relayer le désordre de l'ordre moral ambiant. Rafael Spregelburg y voit une manière de parler de la confusion de sa propre époque dans ce qu'elle porte de terrible mais aussi de prometteur. Ainsi, dans un rapport plus inventif qu'illustratif, Rafael Spregelburd écrit sept pièces indépendantes qui sont reliées subtilement entre elles comme autant de pêchés inédits s'inspirant des originels. Le péché de luxure donne lieu à L'Inappétence, celui d'envie à $L^{\prime} E x$ travagance, celui d'orgueil à La Modestie, celui d'avarice à $L a$ Connerie, celui de paresse à $L a$ Panique, celui de gourmandise à La Paranoïa et celui de colère à L'Entêtement.

La proposition ici consiste à s'attarder sur l'avant-dernière œuvre de la série La Paranö̈ ${ }^{2}$, afin de voir comment et pourquoi le grotesque participe d'une remise en question de la fiction à notre époque. Après un rapide retour sur la perspective grotesque elle-même dans un premier temps, nous vérifierons l'hypothèse d'une œuvre qui recourt ouvertement aux moyens de la fiction classique mais pour mieux en troubler les enjeux et ses lecteurs-spectateurs avec.

\section{Le grotesque}

Dans un premier temps, revenons sur la notion de grotesque qui, malgré sa dimension hautement historique, reste pertinente pour aborder certaines œuvres contemporaines.

Il faut dire que parmi les différents comiques que l'on connait, le grotesque tient une place à part pour relever à la fois du rire et de l'angoisse, $\mathrm{du}$ plaisir et du trouble. Cette conciliation du différent voire du contradictoire semble une constante dans les différentes et historiques versions du concept de grotesque. Nous pensons bien sûr aux figures fantastiques retrouvées dans la Domus Aurea, demeure de l'empereur romain Néron, qui donnèrent son nom au registre à partir du mot de grotte. Ces figures innovèrent par leur étrangeté en étant à la fois minérales, animales, végétales et humaines et inspirèrent les peintres de la Renaissance puis les auteurs jusqu'à aujourd'hui. ${ }^{2} I l$ faut évoquer également le désordre du carnaval médiéval et ses inversions systématiques de valeurs et de hiérarchie qui constituent une autre dimension du grotesque davantage politique. C'est le théoricien et historien

2 La Paranö̈a est écrite dans ses grandes lignes en 2005 à l'occasion d'une résidence en Allemagne puis transformée et réécrite au cours des répétitions pendant deux ans avec les cinq acteurs de la troupe. Elle est jouée en 2007 à Buenos Aires. En France, elle sera traduite et montée en 2009 par le collectif d'acteur du Théâtres des Lucioles 
littéraire Bakhtine qui analyse ce grotesque carnavalesque subversif dans les œuvres de Rabelais, notamment avec Gargantua ${ }^{3}$. Plus proche de nous, la qualification du grotesque selon Baudelaire est particulièrement intéressante dans son Essence du rire ${ }^{4}$ qui, face aux grimaces du clown anglais qu'il à l'occasion de voir à Paris, parle de ce rire comme le comique absolu capable de mêler plaisir et angoisse, rire et horreur. Dans ce sens, Baudelaire nous dit que le comique absolu vient contraster avec les autres comiques de mœurs plus confortables pour le spectateur car ce premier oblige instinctivement sa victime à rire et à brouiller ses repères en termes de moralité:

J'appellerai désormais le grotesque comique absolu, comme antithèse au comique ordinaire, que j'appellerai comique significatif. Le comique significatif est un langage plus clair, plus facile à comprendre pour le vulgaire, et surtout plus facile à analyser, son élément étant visiblement double: l'art et l'idée morale; mais le comique absolu, se rapprochant beaucoup plus de la nature, se présente sous une espèce une, et qui veut être saisie par intuition. Il n'y a qu'une vérification du grotesque, c'est le rire, et le rire subit $[\ldots]^{5}$

Si nous suivons la réflexion de Remi Astruc dans Vertiges grotesques, Esthétique du "choc" comique, (roman-théâtre-cinéma) ${ }^{6}$ qui s'appuie justement sur l'analyse baudelairienne pour aborder des œuvres du XX et $\mathrm{XXI}^{\circ}$ siècle, il s'agit de penser le grotesque comme un " vertige» qui s'impose au lecteur-spectateur:

On pourra donc définir l'effet de grotesque comme ce vertige particulier qui s'empare du récepteur et le déstabilise au moins provisoirement - par l'intrusion d'éléments (images, codes linguistiques,

\footnotetext{
Bakhtine, 1982.

Baudelaire, 1962.

Baudelaire, 1962, p. 254.

Astruc, 2012.
}

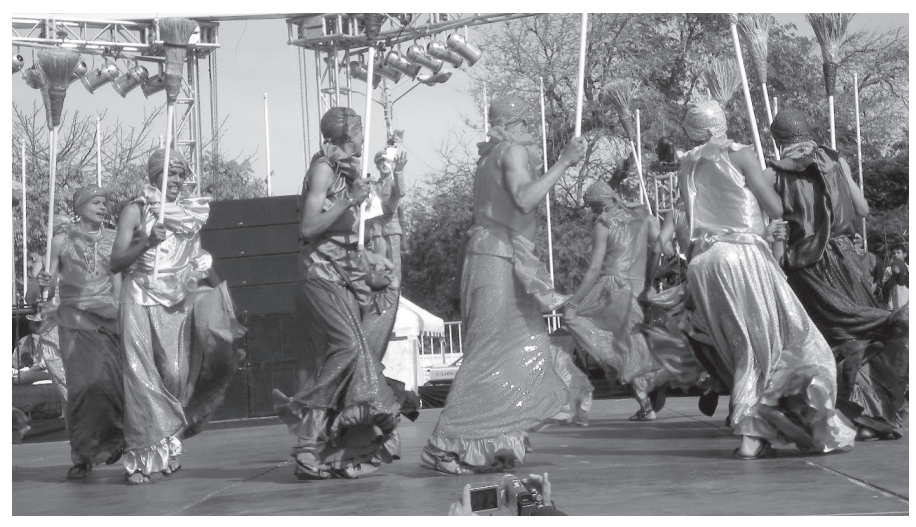

éléments habituellement antagonistes et exclusifs les uns des autres, etc.) qui perturbent sa perception normale et les conditions habituelles du sens? ${ }^{7}$.

Le régime grotesque est pour Rémi Astruc celui de l'invraisemblance, de l'improbable, de l'étrangeté bref de l'impossible réalisé. Il met à l'épreuve depuis la fiction et l'imagination ce qui nous est familier, commun, évident à travers toutes sortes d'hybridations, de dédoublements et autres déformations excessives. Le grotesque ne se réduirait donc pas à une question de forme, de procédés, ni de genre littéraire mais impliquerait une vision du monde alternative.

Rémi Astruc parle en effet ouvertement d'une fonction anthropologique du grotesque avec $L e$ renouveau du grotesque dans le roman $d u X X e$ siècle: essai d'anthropologie littéraire ${ }^{8}$. Dans ce sens, le grotesque serait un outil historique que l'individu et le collectif se seraient donnés pour pouvoir appréhender l'altérité dans ce qu'elle implique de changement, de nouveau, d'inconnu. Le grotesque relèverait forcément d'un moment d'instabilité, d'indétermination, d'indistinction entre des états différents. Cette approche nous semble éclairante au moment d'analyser une œuvre comme La Paranoïa de Rafael Spregelburd en particulier pour développer le questionnement de cette œuvre au sujet de la fiction et sa fabrication.

\footnotetext{
Astruc, 2012, p. 9.

Astruc, 2010.
} 


\section{L'histoire}

Si nous essayons maintenant de nous livrer à la discutable et frustrante tâche de résumer la pièce La Paranoïa, nous le faisons tout en sachant que tout le projet d'écriture réside justement dans la multiplication, l'emboitement et l'embrouillement d'intrigues apparemment indépendantes.

La pièce commence pour nous, lecteur-spectateur, dans une certaine confusion dans la mesure où se succèdent des scénarios a priori totalement indépendants, tant pour leur contexte spatio-temporel que pour leurs personnages. On passe d'un sous-marin russe (ou lituanien nous dit-on) à la maison d'une princesse chinoise séquestrée par des samouraïs japonais au sous-sol d'un laboratoire de chirurgie esthétique vénézuélien. C'est seulement lors de la $4^{\circ}$ scène qu'une intrigue principale semble se distinguer et justifier le désordre fictionnel ambiant. Nous en concluons alors que nous sommes face à une pièce de science-fiction qui se passe en l'an 20000 après JC, dans un système galactique où d'étranges Intelligences gouvernent les autres planètes. Jusque-là, les humains s'en sortaient plutôt bien grâce à leur capacité unique d'inventer des histoires.

Il faut savoir que les Intelligences consomment toute fiction produite par les terriens. Cependant, après avoir épuisé les réserves de livres, de musique, de dvd et même de danse, les Intelligences exigent aux humains une fiction complètement inédite sous peine d'éradication dans les 24 heures. Face au problème, les Opérations Spéciales Terriennes convoquent un

Cependant, après avoir épuisé les réserves de livres, de musique, de dvd et même de danse, les Intelligences exigent aux humains une fiction complètement inédite sous peine d'éradication dans les 24 heures. groupe d'élite, composé d'un astronaute, un mathématicien, une auteure à succès et une G4 (ancienne génération de robot). Pendant toute la pièce, ce groupe s'efforce d'inventer cette fameuse fiction suprême sans succès, capable de répondre à l'intelligence des Intelligences, multipliant les pistes et les techniques mais forcée de les abandonner unes à unes.

Pourtant sur l'écran de projection où sont montrées tant bien que mal les fictions inventées par le groupe, une des histoires prend racine et se développe. C'est l'histoire de Brenda, petite fille vénézuélienne victime des erreurs de la politique commerciale du Venezuela sur le marché de la beauté. Enfermée dès l'enfance pour subir de multiples opérations chirurgicales et ainsi remporter les concours de Miss Monde, Brenda est abandonnée à son sort alors que les opérations dentaires, capillaires et morphologiques ne sont pas terminées quand la mode passe des brunes aux blondes. Pour se venger, elle kidnappe un des chirurgiens et disparait. Entre alors en scène l'inspecteur Lazaro qui va filer l'enquête pour retrouver le chirurgien et découvrir le secret de Brenda.

Il découvrira alors tout le secret de la pièce. Brenda est l'inventrice de cette fiction, des intelligences aux opérations spéciales en passant par le sous-marin russe et l'inspecteur vénézuélien dans son cahier d'écolière. Finalement toutes ces histoires se résume au fantasme de Brenda, dans « un espace vaguement lunaire, ou du moins comme pourrait l'imaginer une petite fille vénézuélienne en convalescence d'une douloureuse chirurgie. " (Spregelburd, 2009, p. 134.)

Nous voyons alors comment l'abîme d'une réflexion sur la fiction et ses limites s'ouvre dans la mesure où cette dernière, la fiction, nous apparait comme monstrueuse et parodiée tout en restant un moyen d'expérimentation revendiqué. 


\section{Une fiction monstrueuse}

L'impression générale est donc celle d'un fourmillement de temps, de lieux, de langues, de personnages et d'histoires qui convoquent un univers symbolique différent à chaque fois. La distribution initiale nous laisse deviner cette multiplication des intrigues:

\section{ACTRICE 1}

Julia Gay Morrison, écrivaine

Lorna Cifuentes, procureur vénézuélienne

Brenda, future Miss Venezuela

Chi-Tsu, bourgeoise chinoise

Alexandra, cliente de salon de coiffure

Mirko La Laitue, artiste transsexuel

Saskja, prostituée lituanienne présumée

Serveuse vénézuélienne

Ludmila, une apparition fugace

\section{ACTRICE 2}

Béatrice,

Alice, remplacement de Béatrice

Astrid, coiffeuse et voyante

Fileuse Chinoise, servante de Chi-Tsu

Secrétaire du président du Venezuela

Zusanna, artiste transsexuel

Iwlowa, prostituée lituanienne présumée

Prostituée vénézuélienne, figurante

\section{ACTEUR 1}

Claus, astronaute

Soldat Nippon 1

Commissaire Kendry Morales

Dr.Barragan, chirurgien

Kwang, mari de Chi-Tsu

Leroy, assistant-coiffeur

Mischi, artiste transsexuel

Mousse du sous-marin lituanien

\section{ACTEUR 2}

Hagen, mathématicien

John Jairo Lazaro, policier vénézuélien

Soldat Nippon 2

Second du sous-marin lituanien

Estéban, une apparition fugace

\section{ACTEUR 3}

Colonel Brindisi

Maria Martha, nonne, sœur de Brindisi

Le Président Chávez

Général Nippon

Capitaine du sous-marin lituanien

Dr Naudi, chirurgien

Patron du Bar /

Policier Vénézuélien, un figurant ${ }^{9}$

Deux actrices et trois acteurs assurent un peu moins d'une quarantaine de rôles entre incarnation sur le plateau et films projetés sue l'écran enregistrés au préalable.

Le prétexte de la science-fiction permet cette mobilité, cette flexibilité mentale pour passer de la Chine féodale, à l'an 20000 au Venezuela contemporain, auxquels se rajoutent dans les dialogues des références à la Norvège, Bierut, la nouvelle Californie, le temps des hiéroglyphes Egyptiens ou encore la culture maya. Le prologue et les 16 scènes (tableaux, fragments, scénarios) multiplient les espaces et les temps en créant des passerelles énigmatiques entre eux.

En effet, en plus d'une multiplicité, l'ensemble relève d'une complexité où des objets, des sujets et des idées entrent en échos d'une scène à l'autre à la manière d'un organisme vivant. Les éléments s'invitent, s'infiltrent et se transposent arbitrairement dans les différentes strates de fiction. Par exemple, des tentures chinoises se retrouvent étrangement dans l'hôtel décadent de la côté uruguayenne où se trouve l'équipe de scénaristes à la manière d'une réminiscence de la première scène, la Barbie fermière de Brenda dans le temps gamma s'invite dans le temps actuel et est adoptée par l'écrivaine à succès. Tous ces procédés laissent deviner qu'il y a une cohérence d'ensemble mais nous maintiennent

$\overline{9}$ Spregelburd, 2009, pp. 7-8. 
dans l'impression que cette cohérence nous échappe.

De fait, la saturation de détails insignifiants dans la description des personnages, le caractère décisif des accidents et des caprices dans la narration qui ne ménagent pas le lecteur et omettent certaines informations, les fausses pistes qui font piétiner l'action sont autant d'éléments qui nous font sentir un certain dysfonctionnement dans la fiction, par rapport à une machine à histoire classique ou, dans le champ du théâtre, à la tragédie. Conditionnant jusqu'à aujourd'hui nos attentes en termes de fiction, Aristote en effet définit la tragédie dans La Poétique, comme la « représentation d'une action menée jusqu'à son terme, qui forme un tout et a une certaine étendue.» (Aristote, 1980, p. 57). La Paranoïa est à peu près tout l'inverse de cette définition et c'est assumé. Les personnages sont eux-mêmes lucides sur ce dysfonctionnement. Comme le signale l'Inspecteur Lázaro:

Quelqu'un me manipule. Quelqu'un manipule ma vie. Par moments, les choses ont du sens, mais tout d'un coup c'est comme si on ... on les réarrangeait. Et

\section{La fiction parodiée}

Chaque personnage convoque à lui seul un genre constitué : la science-fiction qui s'appuie sur d'hypothétiques progrès scientifiques et techniques pour développer une vision $\mathrm{du}$ futur, le policier qui met en scène une énigme qui doit être résolue au cours de l'histoire le plus souvent grâce à une enquête de police, la série b qui est réalisée avec peu de moyens et se transforme fréquemment en série type télé, le mélodrame qui est caractérisé par l'exagération pathétique des effets et un certain manichéisme dans les situations, le film d'horreur qui convoque l'angoisse et la répulsion face à des situations dramatiques, etc. Mais

$10 \quad$ Spregelburd, 2009, p. 78. certaines choses apparaissent n'importe où. Moi-même. Je me réveille ici ${ }^{10}$.

Cette dimension difforme de la fiction atteint encore un autre niveau quand s'opère la mise en abyme et le renversement final des niveaux de fiction au moment où l'équipe découvre que Brenda n'est finalement pas l'invention mais celle qui invente. Les quelques pistes de sens que nous avions réussi à rassembler, guidés par le principe de Science-Fiction se dispersent. Il nous faut rétrospectivement relire l'œuvre pour comprendre les indices que nous avions ratés. Et de fait, un certain plaisir s'installe à pouvoir tisser une logique, même si autre, même si résistante, à pouvoir identifier des choses à la place du chaos premier. La pièce joue donc avec notre endurance et notre curiosité mais aussi avec notre besoin de mettre du sens sur toute chose.

Si le sens général met du temps à se dessiner, voire ne le fait jamais, la profusion de corps, de décor et de discours identifiables occupe dès le début notre logique de reconnaissance. L'œuvre propose un mélange de toutes sortes de genres populaires de la littérature, du cinéma, de la télévision, de l'opéra qui à peine identifiés par le lecteur sont détournés.

les traits empruntés de ces genres semblent tellement grossiers que la parodie ne tarde pas à s'imposer. L'identification du lecteur-spectateur avec les situations est rendue en effet impossible par la dimension volontairement caricaturale et stéréotypée des éléments. Les personnages eux-mêmes dénoncent leur propre médiocrité reconnaissant que "Tout ça est mal écrit, écrit des milliers de fois, c'est du pur cliché. » (Spregelburd, 2009, p. 133).

Cette lucidité tend de fait à déplacer le cliché lui-même vers la question de la redoutable et persistance efficacité de ces scénarios bons marchés. En particulier, Julia, la scénariste de bestsellers, dévoile ainsi la recette d'une histoire destinée avant tout à se vendre: 
JULIA: Mais, qu'est-ce que vous voulez qu'on fasse ? Qu'on invente une fiction avec d'autres catégories, avec des règles que personne ne connait, que nous fassions des marionnettes pour sélénites. Et qui va nous entraîner pour ça ?

COLONNEL: Personne. Faites ce que vous savez déjà faire.

JULIA : Ah bon? Je vais vous dire ce que je sais, je vais vous montrer comment je travaille. Pour que vous compreniez que ce n'est pas de la mauvaise volonté. Vous voulez un succès? Elle s'assoit pour réfléchir. Tous les autres l'observent. Rien ne lui vient. Faites voir, passez-moi ce que vous avez dans ce cartable. Elle fait référence à une petite valise.

COLONEL: Là-dedans ? De quoi avez-vous besoin?

JULIA: N'importe quoi. Ce que vous avez à portée de main.

COLONEL : Une carte. Il la lui donne.

JULIA: Un planisphère. Génial. Elle met le doigt n'importe où. Puis elle le passe à Béatrice. Béatrice, tu pourrais mamplifier ça ? Béatrice monte sur une bicyclette fixe, pédale un peu et projette la carte sur le mur. C'est une partie des Guyanes et des Caraïbes. Qu'est-ce que c'est que ça, comment ça s'appelle ? Guaya...Guaya...?

COLONEL: Venezuela.

JULIA: Venezuela. Voyons... deux ou trois clichés... Caraïbes. Pétrole. Miss Venezuela. Quoi encore ? Elle observe les autres. Quelqu'un veut participer?

CLAUS: Une énigme policière. [...] Une fille vénézuélienne, timide, brune, est prise par l'État... [...] Encore mieux, c'est une Corporation... qui entretient des liens forts avec l'État... une administration parallèle au gouvernement vénézuélien.

JULIA: Bien. La fille est choisie parmi plusieurs petites vénézuélienne...Hm. Ça marche. La petite est née sur un site touristique, disons... Regardant la carte. [...] L'île Margarita. C'est là qu'elle est née. Et dès le début de l'école primaire... [...] on commence à lui faire subir des opérations chirurgicales. Et cette Corporation la prépare pour qu'elle soit Miss Venezuela et par la suite, logiquement, Miss Monde. Fin. Vous voyez, colonel? Je me laisse régir par les clichés et le caprice. Qu'est-ce qui vous fait penser que je vais pouvoir construire pour vous une fiction que vos copines étrangères n'aient pas encore gobée ${ }^{11}$.

Si une première reconnaissance des références qui donnent lieu aux caricatures est aisée, les éléments de parodie en viennent à être eux aussi dénaturés par l'ensemble grotesque qui les juxtapose. En effet, le montage baroque des clichés donne lieu à un tableau qui n'est en rien facile à saisir dans son ensemble mais qui a l'avantage d'ouvrir d'autres terrains de sens.

A titre d'exemple, notre commando d'élite se compose d'un mathématicien clairement limité en calcul mental, d'un astronaute épileptique rescapé d'un accident aérospatial, d'une romancière de bestsellers forcée de s'auto-plagier pour raison commerciale, et enfin d'un robot g4 caractérisé par sa trop grande émotivité. Le tout est chapoté par le Colonel des Opérations Spéciales qui doit se dédoubler en sa propre sœur, une nonne tyrannique, pour imposer son autorité. Nous voyons bien alors que la fiction nous amène à une autre appréhension de la parodie qui se caractérise par l'indétermination grotesque des identités et des corps et qui prône davantage une créativité libérée de l'imaginaire.

Un des éléments privilégiés du brouillage grotesque est la rencontre rendue naturelle de ces mêmes registres populaires que nous venons de citer avec un discours scientifique ou plutôt parascientifique d'une grande complexité. La science-fiction futuriste des Intelligences qui est au cœur de l'œuvre introduit par exemple

11 Spregelburd, 2009, pp. 42-44. 
des histoires extraterrestres parallèles au récit de l'humanité ou des comptages de la temporalité différents se basant sur le modèle du système maya et non pas par années. C'est surtout la course à la fiction suprême, inédite, qui mimerait la complexité du système cognitif des Intelligences qui nous plonge en pleine Théorie du Chaos, des Lois fractales et des Etudes linguistiques. Ici, Hagen le mathématicien s'apprête à expliquer ses recherches effectuées sur les échantillons de pierres cosmiques appelés séfaraton que Claus lui à ramener de son expédition dans le temps gamma:

Moi, je... Je cherche des ressemblances, des répétitions, des liens... Je ne suis pas psychopathe. Je les jette au hasard. Ils tombent. Je relie les fragments entre eux. Je prends des notes... [...] Je fais une nomenclature. [...] Unité Séfaraditique Primarie ab247. [...] Ce n'est pas un «nom ». Le numéro synthétise... le lien... la capacité de celui-ci de tomber près de celui-là... une quantité $\mathrm{x}$ de fois... L'amour pragmatique des mathématiques ne peut se transmettre ${ }^{12}$ !

L'équipe continue ensuite dans son émulsion et traduit les relations mathématiques en mots, ou groupe de mots potentiels, pour ébaucher une nouvelle histoire d'un salon de coiffure qui comme par hasard se recoupe avec l'enquête de l'inspecteur Lázaro. Ainsi, la complexité scientifique n'est pas un but en soi, le lecteur ou spectateur lambda pouvant difficilement suivre les détails. Il semble plutôt qu'au fur et à mesure où la science se développe, elle s'emmêle pour devenir l'équivalent d'une fiction comme les autres, pas moins arbitraire, ni plus décisive.

La science se mêle ici avec des réflexes de registres plus populaires nous laissant l'impression que tout le monde à y gagner. En effet, d'une part la pensée complexe vient remettre de la mobilité dans les stéréotypes et les représentations figées, familière de nos héros de

$12 \quad$ Spregelburd, 2009, pp. 75-74.

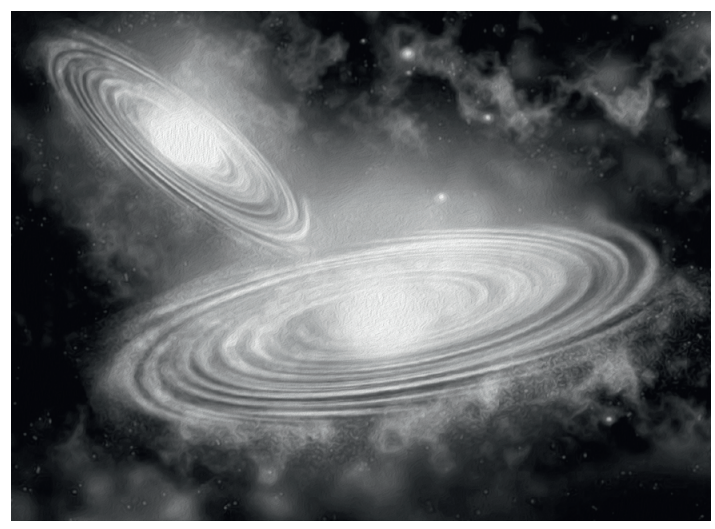

télé. D’autres parts les références culturelles largement partagées réinsuffle de l'envie, du désir face aux enjeux des réflexions scientifiques, métaphysiques ou encore linguistiques. Les deux registres, sérieux et léger regagnent leur potentiel créatif et significatif à travers cette parodie désacralisante.

Ainsi, l'intrigue principale, à savoir la tentative d'inventer une fiction supérieure, nouvelle et infaillible est largement remise en question dans ses conditions de possibilités mêmes. L'usine à fiction dans sa dimension scientifique, commerciale ou populiste est empêchée, déformée, renversée, mise à distance. Cela amène à se demander s'il existe une fiction libre de ces injonctions et plus largement quel peut être, doit être, le rôle de la fiction aujourd'hui.

\section{Une fiction revendiquée}

Au risque de mal ou trop interpréter, il nous semble qu'une scène de la pièce elle-même essaie de répondre à ces questions. Il s'agit de la scène 10 qui se déroule lors d'une vidéoconférence interplanétaire entre les différents groupes d'opérations spéciales. Ces dernières se partagent les informations les plus actuelles au sujet des intelligences et exposent certaines règles pour une fiction réussie:

Règle numéro un : elles n'acceptent pas de premier rôle. Les Intelligences ne reconnaissent pas l'idée du personnage. Elles ne comprennent pas le « je », mais seulement le «nous ». 
Numéro deux. Elles n'acceptent aucun style. [...] Ils disent... que quand les Intelligences voient une chose qui ressemble à une autre, par rapprochement ou par affinité, elles s'ennuient immédiatement.

Numéro trois. Elles n'acceptent pas de hiérarchie. [...] Elles ne supportent pas qu'une chose se prétende plus "importante » que d'autres choses... qui du coup passeraient au second plan. [..] Elles n'aiment pas qu'on leur dise où regarder. [...] pendant qu'elles regardent... elles aiment voir ce qu'il y a à voir, mais elles aiment aussi penser à d'autres choses. [...] Cette règle suppose que l'important ne doit jamais se voir.

Règle numéro quatre. On ne doit pas favoriser l'identification. [..] Elles n'aiment pas identifier quoi que ce soit.[...] Elles n'aiment pas voir ce qu'elles comprennent déjà.

Finalement, la règle numéro cinq dit la chose suivante. [...] ça doit s'adresser au plus grand nombre. [...] Au plus grand nombre... la fiction... ne sert pas si elle satisfait seulement le goût de certaines. Ou de quelques-unes. Majorité13.

Il ne s'agit peut-être là que de règles hasardeuses, capricieuses et contradictoires ou alors, le jeu en partie irréalisable que l'auteur s'est donné pour écrire La Paranoïa. Toujours est-il que ces règles laissent imaginer un horizon de fictions riches et atypiques, inclassables en termes de genre et de lecteurs, bref grotesques.

Brenda n'en serait peut-être que l'expérimentatrice qui rature, griffonne, déconstruit et reconstruit dans son cahier. Brenda qui transcende comme elle peut sa convalescence douloureuse par la fiction. Qui grâce à cette fiction, réinvente ce qui l'entoure, ce qui lui arrive et décide un peu plus de son destin. Quitte à tuer un chirurgien. C'est peut être ça la fiction un moyen de subjectivation parmi d'autres.
La Paranoïa se donne donc pour but de nous projeter dans le futur de l'humanité où règnent la superficialité et la médiocrité afin de mieux réfléchir en creux le pouvoir et la nécessité de la fiction dans nos sociétés. C'est la même idée que développe déjà Rafael Spregelburd quelques années auparavant lors d'un entretien:

Sans fiction, sans altérité, la pensée n'est pas possible. Nous avons oublié à quel point la fiction est importante, le mystère presque sacré du fictionnel. Les enfants le connaissent bien ; quand ils jouent, quand ils mettent un trauma en jeu, en mot, en autre chose, ils exercent un contrôle sur le réel par le langage. Lentement, ce langage tombe dans du sens commun : un ensemble de règles arbitraires, mais qui s'auto-désignent comme uniques. La fiction, depuis sa capacité au caprice, sa force transformatrice, depuis son aptitude surprenante à nous présenter les choses comme si c'était la première fois que nous les voyions (par une sorte de simple expérience existentielle) est une nécessité viscérale dans toute société ${ }^{14}$.

\section{Conclusion}

Il s'agissait ici de penser ensemble les notions de fiction et de grotesque au regard de l'œuvre La Paranoïa qui s'efforce de porter une réflexion plus large sur l'activité fictionnelle en elle-même.

Pour ce qui est du grotesque, limaginaire de Rafael Spregelburd dans La Paranoïa en semble une des incarnations majeures autant pour sa dimension foisonnante que son recours au détournement permanent. Les personnages, plus figures hybrides qu'entités psychologiques, relèvent d'une identité et d'un corps souvent incongrus et incomplets qui, une fois la référence stéréotypée annoncée, se déforment et se troublent. De même, les discours et les situations qui surgissent et

14 Hernández Marisa, 2005, pp. 41-49. 
rapidement digressent empruntent autant aux genres populaires de la télénovela et de la serie b quà la théorie du chaos et aux lois fractales mathématiques. Dans ce mélange étrange de sources et de références (scientifique, historique, d'actualité mais aussi fantastique, surnaturelle et clairement fictive), la tendance parodique ne devient jamais une finalité. Elle est plutôt un point de départ qui est par la suite détourné et démultiplié pour provoquer une surenchère créative et du coup une suspension dans nos habitudes de réception.

Cette suspension laisse justement place à un questionnement au sujet de la fabrique de la fiction qui est à la fois critique et encourageant. A l'instar de l'intrigue dans $L a$ Paranoïa, la fiction est en effet une pratique qui nous caractérise et dont on a besoin en tant qu'humains. Au pire, elles alimentent les dispositifs de contrôle et intègrent les systèmes de consommation, au mieux elles offrent des espaces autres pour nous penser voire nous réinventer autrement. Ainsi la seule utopie que propose peut-être La Paranö̈a, par ailleurs inquiétante sur le futur de l'humanité, c'est cette pratique grotesque de la fiction qui perdure 20000 ans après et qui est notre manière singulière de nous sauver et de conserver la paix.

Finalement, Rafael Spregelburd semble nous livrer à travers cette œuvre son manifeste de la fiction qu'il souhaite collective et plurielle, sans style ni hiérarchisation et toujours à la fois populaire et critique. La fiction se dessine alors comme une expérience vertigineuse, pour reprendre les termes de Rémi Astruc, où le grotesque a toute sa place jusquà aujourd'hui.

\section{Références bibliographiques}

Aristote (1980). La Poétique. Paris: Seuil, collection «Poétique », chapitre 6, 1450 (a 38).

Astruc, R. (2010). Le renouveau du grotesque dans le roman du XXe siècle: essai d'anthropologie littéraire. Paris : Éditions Classiques Garnier.

Astruc, R. (2012). Vertiges grotesques, Esthétique du "choc" comique, (roman-théâtre-cinéma). Paris: Unichamp-Essentiel Honoré champion.

Bakhtine, M. (1982). L'Euvre de François Rabelais et la culture populaire au Moyen âge et sous la Renaissance (1940). Paris: Gallimard.

Baudelaire, C. (1962). «VI. De l>essence du rire et généralement du comique dans les arts plastique » [1855], in Curiosités esthétiques; L'art romantique et autres ouvres critiques, Paris: Garnier.

Hernández, M. (2005). Rafael Spregelburd. Hispamérica 34, n 102.

Spregelburd, R. (2009). La Paranö̈a, trad. Di fonzo bo, M. et Pisani, G. Paris: L'Arche. 